drastically improving the uptake of measles vaccination in the community and by finding more effective prophylaxis for those immunocompromised patients known to have been exposed to the measles virus. If the vaccine uptake in the general population were to be increased to over $90 \%$ as it is in the United States of America, ${ }^{1213}$ as opposed to $50-60 \%$ as in some parts of the United Kingdom, ${ }^{14}$ the herd immunity would be much improved and the risk of contact for those still susceptible would be greatly reduced. Children who are immunocompromised during the period of treatment (which can last up to three years) and for several months thereafter are encouraged to lead as normal a life as possible including attendance at school, though parents and teachers are warned about avoiding contact with infectious diseases. Several recent reports ${ }^{15.17}$ have shown that a major effort by the primary health care and community medicine teams can increase immunisation uptake enormously with subsequent benefit for the community (measles can still cause mortality and morbidity in otherwise healthy children $)^{1819}$ as well as for special groups like immunocompromised patients. Even if the problem of the partial failure of the immunisation programme could be overcome, however, effective prophylaxis would still be needed and a trial of a new high dose intravenous hyperimmune globulin which has been produced by the Scottish National Blood Transfusion Service is being undertaken at the four centres which participated in this study and at the Royal Victoria Infirmary, Newcastle. It is proposed to investigate the use of a newer high dose interferon, possibly in conjunction with the high dose intravenous immunoglobulin, as treatment in complicated cases.

We thank Dr Judith Chessells of the Hospital for Sick Children, Great
Ormond Street, London, for permitting us to include her patients and Mrs Mary McAllister and Mrs Maureen Marshall for secretarial work.

\section{References}

1 Mauer AM. New directions in the treatment of acute lymphoblastic leukaemia in children. $N$ Engl

$\mathcal{F}$ Med 1986;315:316-7.
2 Hughes TW, Kuhn S, Chaudhary S, et al. Successful prophylaxis for Pneumocystis carinii Hughes TW, Kuhn S, Chaudhary S, et al. Suce
pneumonia. N Englf Med 1977;297:1419-26.

3 Hann IM, Prentice HG, Blacklock HA, et al. Acyclovir prophylaxis against herpes virus infections in severely immunocompromised patients: randomised double blind trial. Br Med f 1983;287: 384-8.

4 Mitus A, Holloway A, Evans AE, Enders JF. Attenuated measles vaccine in children with acute leukaemia. Am $\mathcal{F}$ Dis Child 1962;103:413-8.

5 Torigoe $\mathrm{S}$, Hirai $\mathrm{S}$, Oitani $\mathrm{K}$, et al. Application of live attenuated measles and mumps vaccines in children with acute leukaemia. Biken $1981 ; 24: 147-51$.

6 Kay HEM, Rankin A. Immunoglobulin prophylaxis of measles in acute lymphoblastic leukaemia. Lancet 1984;i:901-2.

7 Pullan CR, Noble TC, Scott DJ, Wisniewski K, Gardner PS. Atypical measles infections in children on immunosuppressive treatment. Br Med $\mathcal{J}$ 1976;i:1562-5.

8 Mitus A, Enders JF, Craig JM, Holloway A. Persistence of measles virus and depression of antibody formation in patients with giant cell pneumonia after measles. N Engl $\mathcal{J}$ Med 1959;261:882-9.

9 Jones HE. Immunosuppression and fatal measles. Lancet 1969;ii:1255-6.

10 Anonymous. Measles encephalitis during immunosuppressive treatment. [Editorial.] $\mathrm{Br} \mathrm{Med} \mathrm{f}$ 1976;i: 1552 .

11 Simpson R, Eden OB. Possible interferon response in a child with measles encephalitis during immunosuppression. Scand $\mathcal{Y}$ Infect Dis 1984;16:315-9.

12 Hinman AR, Eddins DL, Kirby CD, et al. Progress in measles elimination. JAMA 1982;247: 1592-5.

13 Katz S. Measles-forgotten but not gone. N Engl f Med 1985;313:577-8.

14 Campbell AGM. Measles immunisation: why have we failed? Arch Dis Child 1983;58:3-5.

15 Ross SK. Childhood immunoprophylaxis: achievements in a Glasgow practice. Edinburgh: Scottish Home and Health Department, 1983:253-7. (Bulletin issued by chief medical officer, 41/5.)

16 Fernandes V, Gill ON. Prevention of measles: vaccine efficacy and potential effectiveness of a vaccination programme on entry to school. Br Med $\mathcal{F} 1985 ; 291: 1685$.

17 Anderson P. Measles immunisation. Br Med f 1985;291:1616.

17 Anderson P. Measles immunisation. Br Med F 1985;291:1616.
18 Miller CL. Deaths from measles. in England and Wales, 1970-83. Br Med $\mathcal{F}$ 1985;290:443-4.

19 Walker E. Reasons for admission to hospital and complications in children with measles. Edinburgh: Scottish Home and Health Department, 1983:300-4. (Bulletin issued by chief medical officer, 41/6.)

(Accepted 24 April 1987)

\title{
Live measles vaccine: a 21 year follow up
}

\author{
CHRISTINE MILLER
}

\begin{abstract}
21 years after receiving Schwarz strain live measles vaccine 4500 trial participants showed a continuing high level of protection compared with those who were unvaccinated. Over the last seven years of the follow up no cases of measles were reported in vaccinated participants who had had close contact with the disease.

Immunity induced by the vaccine seems to survive the challenge of close contact with measles in young children, even after 21 years.
\end{abstract}

\section{Introduction}

In September 1964 a Medical Research Council trial began in England and Wales to assess the immediate ariu long term protection afforded by measles vaccines. Over 36000 children aged between 10 months and 2 years were randomly allocated either to receive live,

\footnotetext{
Public Health Laboratory Service Communicable Disease Surveillance Centre, Colindale, London NW9

CHRISTINE MILLER, BM, MFCM, senior epidemiologist
}

further attenuated measles vaccine alone (9538 children) or four weeks after an injection of killed vaccine (10434) or to remain unvaccinated (16239). Those who had a history of measles were excluded from the trial. Both vaccinated and unvaccinated children (including those ineligible because of illness and those who defaulted from vaccination) were followed up by a nurse at three, six, and nine months to assess clinical reactions to the vaccine and :establish the incidence of measles in each group.

Four previous reports have been published. The first described the design of the trial and covered the initial six months of the follow up.' The second and third reports gave the results at two years nine months ${ }^{2}$ and four years nine months, ${ }^{3}$ respectively, and the fourth reported 12 years after vaccination. ${ }^{4}$ The present report gives the results for a further nine years up to September 1985, 21 years after vaccination.

\section{Subjects and methods}

As previously stated, ${ }^{23}$ the number of participants in the trial was reduced after the first nine months. Over 2000 children from each of the three vaccine groups were lost to follow up when five of the participating local authority areas withdrew from the trial to take part in a community investigation. ${ }^{5}$ In addition, because of an undertaking given at the outset of the trial, vaccination was offered to the control group after the first nine months; 7000 children were vaccinated and were therefore lost to follow up as controls. 
Because of this from 1969 the unvaccinated group was extended to include those whi had defaulted or were ineligible for vaccination in 1964 as well as the original randomly allocated controls; this was considered to be justifiable as the incidence of measles in all three unvaccinated groups was similar. ${ }^{+}$

From October 1965 a yearly postal inquiry was sent to parents, and later to the participants themselves, from the Epidemiological Research Laboratory of the Public Health Laboratory Service. The inquiry asked if the participants had had measles or close contact with measles since the previous year, and the details of any contact were requested. An accompanying letter gave information about the trial's progress. Two further approaches were made if there was no response. Participants from whom no reply was received for three successive years were excluded from further follow up.

For each case of measles reported by a parent or participant confirmation was sought from the general practitioner. If the patient had not been seen by a doctor or if-rarely-the doctor did not reply the case was taken to be unconfirmed. Those who reported an attack of measles that was confirmed by the doctor were no longer followed up; where the doctor gave an alternative diagnosis the participant was informed and remained in the trial. Unvaccinated participants who were vaccinated under the national scheme were also excluded after the date of vaccination.

By 1976 postal contact had been maintained with about 5500 participants in each of the two vaccinated groups and 600 in the unvaccinated group. From 1976 to 1985 the average yearly response to the inquiry was $94 \%$ in the two vaccinated groups and $85 \%$ in the unvaccinated. By September 1985 , when the twenty first year of follow up was complete, the numbers of participants responding in the three groups was 4585 (live vaccine), 4761 (killed and live vaccine), and 296 (unvaccinated)

Vaccines-The freeze dried further attenuated live vaccine was prepared by Glaxo Laboratories from the Schwarz strain of measles virus derived from the Enders-Edmonston B strain (now in use as Mevilin-L, Evans Medical). The killed vaccine (withdrawn in 1968) was prepared by Pfizer Ltd from the Enders-Edmonston B strain.

\section{Results}

\section{ATTACK RATE IN VACCINATED AND UNVACCINATED PARTICIPANTS}

From 1964 to 1969 the average percentage protection given by live vaccine was $91 \%$ in all participants and $94 \%$ in those who reported close contact with measles. ${ }^{3}$ From 1969 , because the unvaccinated group then comprised defaulters and those ineligible for vaccine as well as allocated controls, the percentage protection was no longer calculated. ${ }^{4}$ In the two four year periods 1969-72 and 1972-6, however, the difference in the incidence of measles between unvaccinated and vaccinated participants was highly significant $(\mathrm{p}<0.001)^{4}$; over the last nine years (table I) the incidence in the unvaccinated group was 15 times that in the group injected with live vaccine $(p<0.001)$. Figure 1 shows the yearly incidence of confirmed measles $/ 1000$

TABLE I-Incidence of confirmed cases of measles according to vaccine group 1976-85

\begin{tabular}{lccc}
\hline & & \multicolumn{2}{c}{ Confirmed cases of measles } \\
\cline { 3 - 4 } Vaccine group & No of participant years & No & Rate/1000 \\
\hline Live vaccine & 43955 & 23 & 0.5 \\
Killed and live vaccine & 45546 & 40 & 0.9 \\
Unvaccinated & 3446 & 26 & 7.5 \\
\hline
\end{tabular}

participants in each group during the 21 years. In unvaccinated participants the incidence was highest in the first years of the trial, when the pattern followed that of measles notifications in England and Wales (fig 2). There was no sustained or significant increase in the yearly incidence during the 21 years in either vaccinated group; in the group given live vaccine this remained under 1/1000 participants from 1974.

\section{SEVERITY OF MEASLES}

During the 21 years doctors assessed more cases of measles as being mild in vaccinated than in unvaccinated participants. The difference was highly significant $(\mathrm{p}<0.001)$ between 1964 and 1972, but as reported cases became fewer the difference was no longer significant. During the last nine years only five cases (three of them in the unvaccinated group and two in the group vaccinated with killed and live vaccine) were described as severe, and no complications or deaths were reported. One girl in the group vaccinated with killed and live vaccine developed measles (confirmed serologically) in the fourth month of pregnancy, which continued without incident to the delivery of a normal baby.

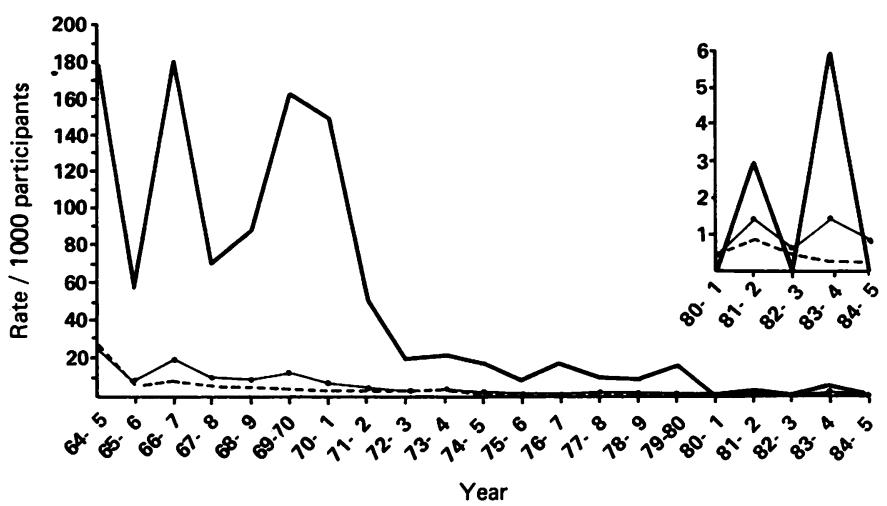

FIG 1-Incidence of measles/1000 participants by vaccine group 1964-85. Unvaccinated. $\bullet-\bullet=$ Killed and live vaccine. $---=$ Live vaccine.

\section{MEASLES AFTER CLOSE CONTACT}

A history of close contact with measles was accepted only when it related to members of the family, children of friends, room mates, or a working contact with children. The incidence of reported contact varied among years but in each year was similar in all three groups. Figure 2 shows the yearly incidences of contact for the three groups combined, together with the number of yearly notifications of measles in England and Wales. The highest incidence of reported contact $(17 \%)$ was in $1966-7$, the year of the last major measles epidemic before vaccination was introduced nationally, when the participants were aged 3-4 years. The incidence decreased to $1 \%$ from 1975 to 1980 , but in 1981 it increased and has since remained at around $3 \%$, although there has been no sustained increase in the number of measles notifications over these years (figure 2).

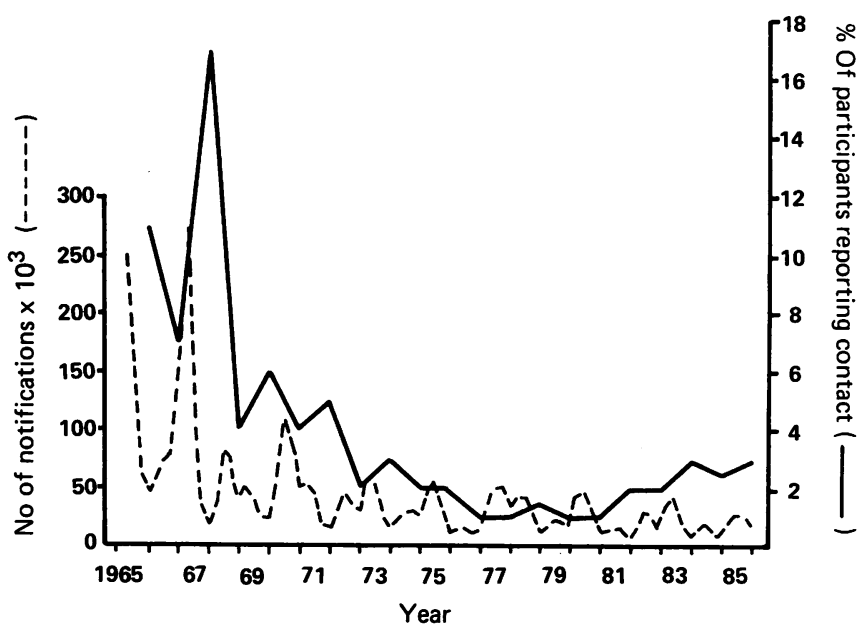

FIG 2-Percentages of participants who reported close contact with measles and number of notifications of measles 1965-85 (England and Wales).

Contact was mainly reported as occurring in the family, in the first seven years from participants' siblings and in the last five years from their own, their siblings', and their friends' children. Recently, contact has also been reported from those working as nurses and teachers.

The difference between the incidences of measles in vaccinated and unvaccinated participants who had been in contact with measles was highly significant $(\mathrm{p}<0.001)$ in each of the three seven year periods of the trial (table II); in the last seven years no cases of measles were reported among participants in the group injected with live vaccine who had been in contact with measles. 
TABLE II-Percentage incidence of measles in trial participants after close contact with measles

\begin{tabular}{lccc}
\hline Vaccine group & $1964-71$ & $1971-8$ & $1978-85$ \\
\hline Live vaccine & $3 \cdot 7$ & $4 \cdot 5$ & 0 \\
Killed and live vaccine & $6 \cdot 5$ & $4 \cdot 9$ & $0 \cdot 7$ \\
Unvaccinated & $79 \cdot 5$ & $47 \cdot 7$ & $11 \cdot 3$ \\
\hline
\end{tabular}

\section{PERSISTENCE OF ANTIBODY IN VACCINATED PARTICIPANTS}

Serum from 47 participants injected with live vaccine, in whom measles haemagglutination inhibition antibody had been detected 10 years after vaccination, was tested five years later, together with the previous specimens. Of the 47 pairs tested, $38(81 \%)$ gave the same results or were within a twofold increase or decrease of each other. In three pairs there was a greater than twofold decrease-to titres of 64,32 , and 32-but none had fallen below 8 . In six pairs there was a greater than twofold increase. In 1974 one participant who had received live vaccine showed no detectable measles antibody. This child developed measles the next year and was not retested in 1979.

In 1974 serum from 37 unvaccinated participants was tested. Despite reporting on entry to the trial and subsequently each year that they had not had measles 25 of the 37 had antibody titres of between 16 and 256 . Of the remaining 12 who did not have the antibody in 1974, seven had had measles by 1979 and three others had been vaccinated; these participants were not retested in 1979.

\section{Discussion}

The 21 years since these children received measles vaccine represents the longest follow up so far reported for a vaccinated cohort of this size. Over the last nine years the size of the vaccinated groups remained remarkably stable, with over 4500 participants in each group still responding every year to the inquiry. The trial shows that live Schwarz strain measles vaccine has continued to give a high level of protection throughout the 21 years, and preliminary data from 1986 extend this to 22 years. The strain and dose of tissue culture are the same as those in two of the three current measles vaccines that now contain improved stabilisers. The combined killed and live vaccine schedule is of only historic interest as no killed vaccine has been used in the United Kingdom since 1968. Nevertheless, this group also showed considerable protection, and no serious atypical measles cases ${ }^{67}$ were reported.

The 23 confirmed cases of measles during the last nine years in participants injected with live vaccine are not an indication of waning protection. The number of cases has not increased over the years and is more likely to be due to the initial failure of the antibody response, which was seen in $4-8 \%$ of children in $1964 .{ }^{1}$ Measles in subjects who had a documented history of vaccination at the age of 12 months or over has been reported both in the United States and the United Kingdom ${ }^{89}$; from the present study there is no such evidence of vaccine failure. Continued susceptibility was shown by 26 confirmed cases of measles in unvaccinated participants over the last nine years as well as by the seven cases among 12 unvaccinated children who did not have antibody in 1974. The antibody study also shows the occurrence of measles without clinical signs, as 25 of 37 unvaccinated participants had antibody but did not have a history of measles.

Some cases of measles in vaccinated participants were described by the doctors as extremely mild, and without a known contact the diagnosis must be doubtful. In participants given live vaccine who reported a close contact with measles, however, an impressive protection provided by the vaccine was seen over the three periods of the trial. No cases occurred after contact in the last seven years, although opportunities for contact evidently increased. This is in contrast with a report from the United States where, in an outbreak of measles in a college, the incidence among vaccinated students increased with the time elapsed since vaccination"; this may, however, have been partly owing to poorer documentation for older students..$^{10}$ It must be remembered that, unlike in the United States where measles is now a noteworthy event, measles in England and Wales remains endemic, with around 90000 cases notified a year. There is therefore ample opportunity for antibodies induced by vaccine to be boosted, and in the small sample of vaccinated participants tested 10 and 15 years after vaccination six of 47 showed evidence of this.

In our trial the number of cases that doctors assessed as being severe decreased as participants grew older, and there have been no deaths since two unvaccinated children died in the first six months.' This again differs from experience in the United States, where, in an outbreak of 128 cases in college students, three deaths from respiratory complications occurred. ${ }^{11}$

The history of close contact with measles reported by participants over the years shows an interesting pattern. The decreasing incidence of contact as the children grew up was to be expected, as measles is still most prevalent in the 1-4 year age group, with whom 12-16 year olds mix less. The increased contact reported as the participants reached 18-20 years, however, shows that, as with rubella, the risk of infection in those susceptible is related to age, with children in the family being the most common source. It is reassuring that after 21 years immunity induced by the vaccine seems to survive the increasing challenge of close contact with measles in young children, because unless acceptance of the vaccine in the United Kingdom increases appreciably from its current level of $60-70 \%$ this chailenge is likely to continue.

The study was performed under the auspices of the measles vaccine subcommittee of the Committee on Development of Vaccines and Immunisation Procedures (chairman Professor J E Banatvala). The subcommittee is grateful to Mrs E Burns and Mrs D Moffatt for their work in maintaining the high level of follow up.

\section{References}

1 Measles Vaccine Committee. Vaccination against measles: a clinical trial of live measles vaccine given alone and live vaccine preceded by killed vaccine. A report to the Medical Research Council. BrMed J 1966;i:441-6.

2 Measles Vaccine Committee. Vaccination against measles: clinical trial of live measles vaccine given alone and live vaccine preceded by killed vaccine. Second report to the Medical Research Council. BrMed $\mathcal{F} 1968$;ii:449-52.

3 Measles Vaccine Committee. Vaccination against measles. Clinical trial of live measles vaccine given alone and live vaccine preceded by killed vaccine. Third report to the Medical Research Council. Practitioner 1971;206:458-66.

4 Measles vaccine subcommittee of the Committee on Development of Vaccines and Immunisation Procedures. Clinical trial of live measles vaccine given alone and live vaccine preceded by killed vaccine. Fourth report to the Medical Research Council. Lancet 1977; ii:571-5.

5 Sutherland I, Fayers PM. Effect of measles vaccination on incidence of measles in the community. BrMed F 1971;i:698-702.

6 Fulginiti VA, Eller JJ, Downie AW, Kempe CH. Altered reactivity to measles virus. JAMA 1967;202:1075.

Haas EJ, Wendt VE. Atypical measles 14 years after immunization. JAMA 1976;236: 1050.

8 Walker D, Carter H, Jones IG. Measles, mumps, and rubella: the need for a change in immunisation policy. $\mathrm{Br}$ Med $\mathcal{F}$ 1986;292:1501-2.

9 Centers for Disease Control. Measles outbreak among vaccinated high school students-Illinois. MMWR 1984;33:349-51.

10 Hull HF, Montes JM, Hays PC, Lucero RL. Risk factors for measles vaccine failure among immunized students. Pediatrics 1985;76:518-23.

11 Centers for Disease Control. Multiple measles outbreaks on college campuses in Ohio, Massachusetts, Illinois. MMWR 1985;34:129-30.

(Accepted 29 April 1987) 